\title{
Towards Standardization of Sampling Methodology for Evaluation of Soil Pollution in Nigeria
}

\author{
${ }^{* 1}$ OKUNADE, I. OLUSOLA; ${ }^{2}$ OKUNADE KEHINDE AISHA
}

\author{
${ }^{1}$ Centre for Energy Research and Training, Ahmadu Bello University, P.M.B. 1014, Zaria, Nigeria, \\ ${ }^{2}$ College of Environmental Sciences,Kaduna Polytechnic, Kaduna, Nigeria.
}

\begin{abstract}
This article proposes the procedure that may be adopted for comparable, representative and cost effective, soil sampling, and thereafter explores the policy issues regarding standardization of sampling activities and analytical process as it relates to soil pollution in Nigeria. Standardized sampling and analytical data for soil pollution will be required for comparative analysis, extrapolation of results to other areas and development of threshold values for risk assessment and soil quality evaluation. For the purpose of initiating the process of standardization of soil sampling, the paper considers the specification for designing, planning and sampling polluted sites and development of methodological aspects such as sampling scale, sample density, sampling coordinate, sampling depths, composite samples, sample pretreatment, quality control etc. The various aspects of soil sampling, which require harmonization, that is a prerequisite for the establishment of National guideline for soil pollution are also enumerated. @JASEM
\end{abstract}

The analytical process for investigating soil pollution may be classified into four stages. These include preliminary investigation, sampling and sample pretreatment, sample analysis and lastly data analysis and interpretation. Among these four stages, greatest importance should be attached to the sampling and sample pretreatment phase. This is because conclusions about an investigated site are often deduced from the concentration of contaminants in the representative soil samples collected during the sampling and sample pretreatment phase, since such analytical data accurately reflects the actual status of the site. In addition, errors in sampling and sample pretreatment, which often exceed analytical error, (Ramsey and Argyraki, 1995) can have detrimental influence on the final results of a soil investigation.

The process of obtaining representative sample of soil from a contaminated site is often faced with a lot of difficulty. This is because external contaminants and radionuclides entering a soil body through waste disposal, wet or dry deposition behave differently in each soil type depending on its absorption properties, texture, density, humidity, geological setting of the site, type and population of microorganisms etc, thereby leading to variability in their spatial and depth distribution. Thus there is always variation in the distribution of pollutants within the site being investigated and within the soil samples collected. In order to attain representative sampling, an efficient sampling and sample pretreatment strategy based on the observed variability of the site, variables that are introduced during sampling, scope of the investigation, and the desired precision must be employed. It should be noted that the accuracy of analytical results for the count samples would not lead to correct conclusion, if they do not represent the site in such a way as to meet the objectives of the investigation. Reproducibility of the results is also important as it allows extrapolation of analytical data to other areas and also comparison of analytical results for soils from different parts of the Country. Thus there is a need to develop standardized and consistent sampling procedures for the evaluation of soil contamination. This can be achieved through harmonization of all the steps involved in the sampling and analytical process throughout the country to minimize errors, deviations and uncertainties that may arise since soil quality standards are always defined on the basis of appropriate standardized sampling guidelines. Such guidelines have to specify the general approach and sampling strategy as well as critical methodological details, which should be flexible in order to allow for realization of individual project objectives and adaptation to actual situation encountered during the fieldwork. The analytical results obtained from such standardized sampling serves as input for development of threshold values for soil contaminants throughout the Country.

\section{PROPOSED METHODOLOGY}

Preliminary Investigation: Preliminary Investigation involves collation of background information about the site under investigation usually from literature or correspondence. Information required includes site location, soil type and climate; industrial activities, fertilizer and pesticide application; wastewater, hazardous wastes and particulate emissions. The disposal practices for the various site contaminants should also be documented. The collated data is usually validated using the data collected during reconnaissance survey of the area. (Nortcliff, 2001). It should however be noted that the preliminary investigation might require limited amount of sampling, preferably surface soil samples and water samples. The information collected during this stage 
of the investigation provides for preliminary risk assessment and determination of scope and methodology for design of sampling plan that will be used for detailed investigation.

Development of Conceptual Model for soil sampling: A conceptual model is used to develop hypothesis regarding the location, and movement of contaminants of interest at the site and potential impacts that may occur to human health, the biota and the environment, Figure 1 shows a typical conceptual model for soil contamination studies. The development of a site specific conceptual model involves collation of sufficient information to identify sources of the contamination, determine the nature and extent of the contamination, identify the dominant fate and transport characteristics of the site, specify potential exposure pathways, and identify potential receptors that may be impacted by the contamination. Historical data of a site can be used to develop conceptual model hypothesis, particularly with regard to the fate and transport characteristics of the site. The conceptual models will also be used to identify the critical decisionmaking parameters for which data needs to be gathered and the uncertainty associated with these parameters. Site conceptual model also serves the purpose of guiding the analyst during sampling and analysis. Site conceptual model hypothesis may also be revised based on findings during sampling. If your site is simulated using analytical and numerical models, you will use the conceptual scenario elements as a reality check and guide. The development of a site specific conceptual model is a critical component in risk-informed cleanup. In any conceptual scenario, the site under investigation must be examined in light of four important elements including nature and extent of the source, dominant fate and transport chrarcteristics, potential exposure pathways and receptors that may be impacted.

Design and Planning of Soil sampling Programm: The basic information obtained from the preliminary investigation should serve as a guide for designing the sampling plan, which is a basic requirement for quality assurance and quality control in the implementation of fieldwork. The sampling plan should provide a detailed description of tasks, objectives, results of the preliminary study of the site under investigation and be able establish a pollution hypothesis. (Wagner et al, 2001). The design of the sampling plan should take into consideration the manner of land use, climate, vegetation, soil type or texture, fertilization effects and sources of pollutants. In addition, it has to predetermine the sampling time, sampling depth, sampling grid or pattern, and the number of locations to be sampled. In order to stratify the sampling site into representative sampling units, uniform areas with identical vegetation from the same land use should be identified. Where there are differences in soil types or textures, the identified areas should be further divided into more sampling units. 1 composite sample is sufficient for 1ha, but for larger areas more composite samples will be required. In order to obtain more information about the site under investigation it is also essential to obtain a depth distribution of pollutant for each sampling unit. For this purpose, the choice of sampling depth will depend on the use and condition of each sampling unit (Bacon et al, 2001). A fixed depth may be used for cultivated (disturbed) soils since the soils are relatively uniform. In some cases, several depths of a disturbed soil need to be sampled especially for consideration of the pathways through which contaminants migrate and their potential effects on ground water supply. On the other hand, undisturbed soil must be sampled through several depths to identify the elemental concentrations associated with each depth in the depth profile. This technique only specifies the general principles involved in spatial and depth sampling. It has not taken into consideration unforeseen circumstances during the actual sampling, such as presence of hot spots, where there is evidence of pollution as a result of observed changes in the physical appearance of soil at that spot. Thus, there should be allowance for alteration of the sampling plan, which should only be authorized by accredited personnel to adapt the sampling strategy to actual situation in the field.

Sampling Strateg: Ideally, collection of representative samples from each sampling unit identified in the sampling design will require collection of several samples, which will be subsequently combined together to form a single composite sample weighing about $1 \mathrm{~kg}$. Cameron et al (1971) had established that 20 individual subsamples bulked into a single composite are sufficient to provide a true mean concentration of a test area and that the size of the area had no serious effect on the accuracy obtained in forming a composite sample. If however, sampling time and cost are likely to constitute constraints, it is sufficient to use between 12 and 15 sub-samples to obtain a composite sample for a particular sampling unit (Bacon et al, 2001; Aichberger and Back, 2001). The process of taking the required number of subsamples may be based on sampling at identified positions along a $\mathrm{W}$-shaped or $\mathrm{X}$-shaped pattern (Aichberger and Back, 2001). Alternatively, sampling could be done within the grid pattern of approximately equal areas formed within the sampling unit and various sampling positions 
selected using the random number table (Theocharopoulos et al, 2000). The latter sampling pattern is always preferred since the former is prone to bias towards the center of the area. Criteria for selecting alternative sampling position in situation whereby the selected sampling position could not be reached or provide the desired samples, should also be established. In a situation where prevailing condition at the site necessitates alteration of the sampling plan, authorization must be sought from qualified personnel and alternative procedure agreed upon must be clearly documented and explained in the sampling plan. Thus, a good sampling strategy should give room for flexibility since the actual sampling must be adapted to real situations at the sampling site, which must be considered based on its observed characteristics.

Sample Collection: The number of samples collected depends on the sampling approach adopted. In the case of grid sampling, sub-samples should be collected at each grid node, irrespective of the grid size. In a situation whereby a hot spot is encountered at a grid node, adjoining grid cells should be more thoroughly sampled to define areas of contamination. Five aliquots from each grid cell situated from the sides of the cell plus one at the grid center are sufficient for grid cell of $30 \mathrm{~m} \times 30 \mathrm{~m}$ size. If the grid size is greater than $30 \mathrm{~m} \times 30 \mathrm{~m}$, then the number of aliquots per grid size should be increased to nine. If the sample collection is limited to surface samples alone then a smaller grid size of $30 \mathrm{~cm} \times 30 \mathrm{~cm}$ should be used. Instead of grid sampling, aliquots may be collected from the various sampling points identified on the "X" or "W" shaped pattern in each of the representative sampling units as the case may be.

The sampling tools usually employed include bucket auger, core auger, trowel, and PVC cylinder. Other sampling tools employed in soil sampling include Spade, knife and forks that are made of stainless steel (Baize and Sterckeman, 2001). In general, sampling equipment that can collect the necessary sample quantity down to a certain depth must be available. In arable land, the ploughed layer, which is normally $0-20 \mathrm{~cm}$ depths, is sampled using the auger. In the case of forest ecosystem, consideration must be given to the litter horizons where considerable amounts of heavy metals tend to accumulate due to the microbial decomposition of litter. Thus it is usual to sample the O- horizons ( 0 $3 \mathrm{~cm}$ and $3-7 \mathrm{~cm})$ and Ah-horizon $(0-8 \mathrm{~cm})$. In the case of grassland, $0-10 \mathrm{~cm}$ and $10-20 \mathrm{~cm}$ or $0-15 \mathrm{~cm}$ and $15-30 \mathrm{~cm}$ depths need to be sampled depending on the size of the auger that is available. In some cases, sampling is done from a pit dug at the sampling site, so that depth profiles in the range of $15 \mathrm{~cm}-140 \mathrm{~cm}$ (Baize and Sterckeman, 2001) can be sampled. This is particularly the case for urban soils, which are often characterized by the natural local pedogeochemical background and contaminated parent material.

Immediately after sampling from each unit, distilled water should be used to clean all sampling tools in order to prevent cross contamination. The aliquots from each grid size are subsequently combined into a single sub-sample. Depending on the budget and other considerations, 12 to 15 sub-samples from each sampling unit may be further combined into a composite sample weighing about $1 \mathrm{~kg}$ in a polyethylene container, after removal of extraneous material such as pieces of glass, twigs, leaves etc, which are not vital to the characterization of the samples. Compositing of soil samples has the effect of averaging the concentration of the contaminants over the number of sampling points and reducing the number of samples to be analyzed. The homogenized composite samples are subsequently transferred into a clean plastic or glass containers and appropriately labeled and stored in the dark.

Sampling Records: The sampling record is also an important part of the quality control system for soil sampling. The sampling record should generally document the conduct of the sampling operation, special features of the site under investigation, observed characteristics of objects sampled, unforeseen circumstances that are capable of influencing the quality of the samples and deviations from the sampling plan. In addition, the samples should be systematically listed, appropriately described and labeled to allow for their adequate handling, prevent mistaken identity and to achieve optimal repeatability in soil sampling. Thus a detailed sampling record should contain information such as sampling date, site identification, weather condition, land use unit, vegetation, size of the sampling unit, sampling points, sampling depth, sample description, sample label, special features during the drilling and the sample retrieval from the equipment, transportation and sample storage condition. Additional documentation includes evidence of fertilizer application at the site, identified contaminant, deviation from the sampling plan, explanation for alteration of sampling plan and name, address and signature of the personnel who conducted the sampling.

Sample Pretreatment: Individual composite samples should be air dried at room temperature $\left(25^{\circ} \mathrm{C}\right)$ on a plastic tray until constant weight is achieved. 
Typically, 3-4 days are sufficient for this purpose. The samples are subsequently disaggregated with a roller, or alternatively crushed and then sieved through a $2 \mathrm{~mm}$ nylon mesh to obtain uniform particle size distribution. In order to provide uniform distribution of contaminants and to ensure that the resulting sample is representative of the total soil sample collected, the sample should be shaken for about $24 \mathrm{hrs}$ and milled to less than $250 \mu \mathrm{m}$ particle size with a mill made of tungsten carbide ring mill in the case of mineral samples and ball mill in the case of H-horizons (Satre et al, 2001). Sub-sampling should then be done by quartering after an additional homogenization. Finally aliquots are taken for further preparation or treatment before analysis.

\section{DISCUSSION}

The need for a coordinated environmental sampling for the survey, assessment and remediation of contaminated sites in Nigeria has necessitated standardization of soil sampling and other analytical steps to ensure comparability of data obtained by different investigators. Indeed comparable sampling and analytical data for soil pollution allows extrapolation of the result of an investigation to similar regions. In addition, it provides the basis for the development of threshold values and environmental risk assessment for soil contaminants. Therefore the methods chosen for the different steps in the sampling and analytical process must undergo the highest possible degree of standardization. In addition, the selected methods should be flexible, easy to handle, reliable as a routine method and part of an evaluation system.

The standardization process should however take into consideration the fact that the objectives of a soil investigation usually determine the sampling strategy and the scope of the investigation. The sampling strategy prescribes size of the sampling unit and number of samples to be collected based on the variability of the investigated parameter, since it must guarantee that the sample is representative of the whole. Since the requirements of a sampling strategy are defined by the objectives of the investigation and characteristics of the site under investigation, it will be difficult to establish suitable strategy for all possible objectives. Therefore, the standardization policy should give room for flexibility in the conduct of sampling operation. This should however be based on the premise that experienced and qualified personnel with the required authority to alter the sampling plan will be involved in the conduct of sampling.

In addition, spatial heterogeneity has to be considered in the course of soil sampling and also in order to limit the number of samples to be analyzed and save cost, composite samples will be required. The collection of composite samples, which entails stratification into various sampling units based on land use, vegetation, presence of hot spots, soil texture etc and fixing of the corresponding sampling depth is expected to be an important component of the standardization policy. Furthermore, sample pretreatment results in variability sources that may cause comparability problems. This is especially the case for samples containing hard bedrock fragments, plant litter and particles size fraction larger than $2 \mathrm{~mm}$ mesh size. Splitting or sub-sampling the sample prior to analysis can also be a serious source of variability especially when mechanical devices using vibrations are involved (Kunt and Muntau, 1994) and may hence also cause comparability problems.

Therefore, in the light of the issues enumerated above, the following aspects of sampling that often results in deviations in the results obtained by various investigators must be considered in order to begin standardization of soil sampling in Nigeria. These include sampling strategy, sample pretreatment, and quality control. Standardization of sampling strategy should be considered from the view point of setting criteria for selecting sampling units, sampling scale, sampling depths, sampling density, single samples, composite samples, sampling pattern, sampling coordinate, sampling technique within a sampling unit, sample spacing, number of samples in a composite, surface and depth sampling. The standardization process for sample pretreatment should also set the criteria for segregating part of the samples and for sub-sampling the resulting samples to obtain count samples. Various aspects of quality control such as sampling tool, transportation, containerization, storage, sampling records, accreditation of institutions to perform soil sampling and qualification of the sampling personnel should also be considered in the standardization process.

It should be emphasized here that the proposed standardization assumes that the analytical techniques for soil analysis are already standardized and that the uncertainties that may arise during analysis are kept to a minimum. If the analytical technique should result in high uncertainty as a result of complex sample preparation procedures and also produce results that are characterized with low precision, variability between samples will be suppressed by the variability of the analytical method or analytical procedure, thereby leading to erroneous interpretation and conclusion. Thus the chosen analytical technique should be simple, 
reliable, reproducible and characterized with high precision. In addition, the technique should support minimum sample preparation steps in order to minimize uncertainties that may arise. Furthermore, quality of the resulting data should be assured through standard operating procedures, proficiency tests, good laboratory practice and continuous quality control programme based on certified reference materials.

\section{REFERENCES}

Aichberger, K; Back J. (2001). 'An Austrian Soil Sampling Procedure Tested in a Field Study (CEEM-Project)'. Science of Total Environment, 264, 175-180.

Argyraki, A. Ramsey, MH; Thompson M. (1995). 'Proficiency Testing in Sampling: Pilot Study On Contaminated Land'. Analyst, 120, 27992804.

Baize, D; Sterckeman, T. (2001). 'On the Necessity Of Knowledge of the Natural PedoGeochemical Backround Content in the Evaluation of the Contamination of Soils by Trace Elements'. Science of Total Environment, 264, 127-139

Bacon, JR; Hudson, G. (2001). 'A Flexible Methodology for the Characterization of Soils: A Case Study of the Heavy Metal Status of a Site at Dornach'. Science of Total Environment, 264,153-162

Brumelle, S, Nemetz, P.; Casey, D. (1984). 'Estimating Means and Variances: The Comparative Efficiency of Composite and Grab Samples'. Environmental Monitoring and Assessment. 4, 81-84.

Burgess, TM; Webster, R (1984). 'Optimal Sampling Strategy for Mapping Soil Types. Distribution of Boundary Spacings'. Journal of Soil Science, 35, 641-654

Cameron, DR, Nyborg, M, Toogood, JA and Laverty, DH (1971). 'Accuracy of field sampling for soil tests'. Canadian Journal of Soil Science, 51, 165-175

Khunt, G; Muntau, H. (1994). 'Euro-soils: Identification Collection Treatment
Characterization'. European Commission Special Publication 1, 60-68

Fons, J. Sauras, T. romanya, J ; Vallejo, VR (1997). 'Sampling Strategies in Forest Soils'. Annals of Science, 54, 493-499

Hammer, RD, Ralph, O and Russel, JL (1987). 'Temporal and Spatial Soil Variability on Three Forested Land Types on the Mid-Cumberland Plateau'. Soil Science Society of American Journal, 51, 1320-1326.

Jackson, KW, Ian, WE; Michael, SW (1987). 'Stratified Sampling Protocol for Monitoring Trace Metal Concentration in Soil'. Soil Science 143(6), 436-443

Nortcliff, S. (2001). 'Sampling and PretreatmentSome Observations from the United Kingdom'. Science of Total Environment, 264,163-168

Ramsey, MH Argyraki, A; Thompson, M. (1995). 'Estimation of the Sampling Bias Between Different Sampling Protocols on Contaminated Land'. Analyst, 120, 1353-1356

Ramsey, MH, Taylor, PD; Lee, JC (2002). 'Optimized Contaminated Land Investigation at Minimum Overall Cost to Achieve Fitness for Purpose'. Journal of Environmental Monitoring, 4(5), 809-814

Rubio, R: Ure, AM (1993). 'Approaches to Sampling and Sample Pretreatments for Metal Speciation in Soils and Sediments'. International Journal of Environmental Analytical Chemistry, $51,205-217$

Sastre, J, Vidal, M, Rauret, G; Sauras, T. (2001). 'A Soil Sampling Strategy for Mapping Trace Element Concentration in a Test Area'. Science of Total Environment, 264, 243-257

Theocharopoulos, SP, Wagner, G, Sprengart, J. Mohr, ME, Desaules, A. Muntau, H. Christou, M; Quevauviller, P. (2001). 'Comparative Soil Sampling in the Dornach Site (Switzerland) for Soil Three Dimensional Pollution Description'. Science of Total Environment, 264, 63-72

Watson, KK; Lees SJ (1975). 'A Procedure for the Sampling and Testing of Large Soil Cores'. Soil Science Society of American Journal, 39, 589590 